\title{
¿Se Puede Evaluar a Niños Rurales con WISC-V? Explorando la Invarianza Factorial de la Inteligencia en Chile
}

\author{
Can Rural Children Be Tested with WISC-V? Exploring the Factorial Invariance of \\ Intelligence in Chile
}

\author{
Marcela Rodríguez-Cancino ${ }^{1}$, María Beatriz Vizcarra ${ }^{2}$ y Andrés Concha-Salgado ${ }^{3}$
}

\begin{abstract}
Resumen
El uso adecuado y ético de test psicológicos implica la revisión de diversas evidencias de validez, dentro de las cuales el examen de la invarianza factorial, permite establecer la equivalencia y/o explorar la imparcialidad de los instrumentos cuando estos se utilizan en diversos grupos culturales. En Chile, la Escala Wechsler de Inteligencia para Niños (WISC) se ha utilizado de manera masiva, tanto en población urbana como rural, a pesar de no contar con estudios sobre su ajuste psicométrico en este último grupo. Con una muestra de 480 escolares, el presente estudio examinó la invarianza factorial de la Escala WISC-V, verificando si los constructos medidos son equivalentes y no varían en función de la procedencia urbana o rural de los niños. Los resultados obtenidos permiten aceptar el nivel de invarianza configural y métrica parcial. Se discuten las implicancias teóricas y prácticas del uso de esta escala en población rural chilena.
\end{abstract}

Palabras clave: WISC-V, invarianza factorial, población rural, población urbana, Chile

\begin{abstract}
The proper and ethical use of psychological tests implies the review of various validity evidences, including the examination of factor invariance, which allows establishing the equivalence and/or exploring the impartiality of the instruments when they are used in different cultural groups. In Chile, the Wechsler Intelligence Scale for Children (WISC) has been used massively in urban and rural populations, despite the lack of studies on its psychometric adjustment in the rural group. With a sample of 480 schoolchildren, the present study examined the factor invariance of the WISC-V Scale, verifying if the constructs measured are equivalent and they do not vary depending on the urban or rural origin of the children. The results obtained allow accepting the level of configural invariance and partial metric invariance. Theoretical and practical implications of the use of this scale in Chilean rural population are discussed.
\end{abstract}

Keywords: WISC-V, factor invariance, rural population, urban population, Chile

Esta investigación fue financiada por la Dirección de Investigación de la Universidad de La Frontera, a través del Proyecto DIUFRO INI17-0016.

Agradecimientos a: (a) Equipo de Ayudantes de Investigación: Claudia Caro Vallejos, Catalina Becerra Muñoz; Francesca Beneventi Balocchi, Laura Lucero Valdebenito, María Soledad Matus Muñoz, Belén Salinas Rehbein, Javiera Solano Montero y Jorge Velásquez Ayala. (b) Equipo de Investigación del Centro de Desarrollo de Tecnologías de Inclusión de la Pontificia Universidad Católica de Chile (CEDETi-UC)

\footnotetext{
${ }^{1}$ Dra. en Psicología, Docente Departamento de Psicología. Universidad de La Frontera, Calle Montevideo 0830, Temuco, Chile. Tel.: +56452325605. Correo: marcela.rodriguez@ufrontera.cl, https://orcid.org/0000-0003-2012-9701 (Autor de correspondencia)

${ }^{2}$ Dra. en Educación, Profesora Asociada, Departamento de Psicología. Universidad de La Frontera, Calle Montevideo 0830, Temuco, Chile. Tel.: +56452325605. Correo: maria.vizcarra@ufrontera.cl, https://orcid.org/0000-0002-7052-7882

${ }^{3}$ Doctorando en Psicología, Docente Departamento de Psicología. Universidad de La Frontera, Calle Montevideo 0830,Temuco, Chile. Tel.: +56452325605. Correo: andres.concha@ufrontera.cl, https://orcid.org/0000-0002-9374-7095
}

Revista Iberoamericana de Diagnóstico y Evaluación - e Avaliação Psicológica. RIDEP · №60 · Vol.3 · 117-131 2021

ISSN: 1135-3848 print /2183-6051online 


\section{Introducción}

Los Estándares para Pruebas Educativas y Psicológicas (AERA, APA, \& NCME, 2014) exponen claramente la relevancia de explorar la imparcialidad de los test de uso masivo, como un aspecto fundamental de la validez. Dentro de esto, el análisis de invarianza permite establecer el grado en que un instrumento se encuentra libre de sesgo de medición, lo que respalda la interpretación y uso de sus puntajes en un contexto y población definida (ITC, 2017; McGill et al., 2020, Pedrero, 2019)

Según Elosua (2005) la invarianza de medida "se define con relación a un grupo o forma de un test, de tal modo que el significado formal y sustantivo de la medición es independiente respecto a ello" (p. 356). Esta autora afirma que sería erróneo comparar las puntuaciones en un constructo que no tuviera la misma representación para los individuos que pertenecen a dos grupos culturales, al igual que es incorrecto interpretar, de una sola forma, los resultados de un test si su aplicación en dos contextos podrían vincularse a errores de medida diferentes.

De acuerdo con Pedrero (2019) el objetivo de los análisis de invarianza es establecer si las relaciones entre los ítems de un instrumento permiten su comparación entre distintos grupos de interés. Para este autor la invarianza de la medición se conceptualiza como una "serie de niveles progresivos que van desde nula invarianza y por lo tanto mayor sesgo de medición hasta niveles estrictos de invarianza en donde no existiría sesgo de medición" (p. 276).

Los pasos tradicionales en la evaluación de niveles de invarianza son: a) Invarianza Configural (invarianza de forma del modelo); b) Invarianza Métrica (equivalencia de las cargas en los factores, entre los grupos comparados, también llamada invarianza débil); Invarianza de Escalar (equivalencia de los interceptos de los ítems entre los grupos comparados, o invarianza fuerte) e Invarianza Residual (explora la equivalencia de los residuos de los ítems entre los grupos, conocida como invarianza estricta) (Putnick \& Bornstein, 2016; Vandenberg \& Lance, 2000).

Siguiendo estas orientaciones el presente estudio aborda el análisis de invarianza factorial de la Escala Wechsler de Inteligencia para niños, quinta edición (WISC-V) según la variable procedencia urbano o rural de los evaluados.

WISC-V es un instrumento clínico para la evaluación del funcionamiento cognitivo de niños, niñas y adolescentes (NNA) de entre 6 y 16 años de edad (Flanagan \& Alfonso, 2017; Rosas \& Pizarro, 2018; Rosas et al., 2021; Wechsler, 2014). Esta escala, ampliamante utilizada a nivel mundial, ha sido traducida, adaptada $\mathrm{y}$ estandarizada en diversos países (Chen, Zhang, Raiford, Zhu, \& Weiss, 2015; Coronel, Lacunza, \& Contini, 2006; Flanagan \& Alfonso, 2017; McGill, Ward, \& Canivez, 2020; Weiss, Saklofske, Holdnack, \& Prifitera, 2019).

Las primeras versiones de las escalas Wechsler estaban organizadas en dos medidas de $\mathrm{CI}$ independientes: CI verbal y CI manipulativo y un CI total que incluía la escala completa (Flanagan \& Alfonso, 2017; Niileksela \& Reynolds, 2019). Según Brenlla (2013) Wechsler consideraba que la inteligencia se componía esencialmente de dos factores, razón por la cual sus test incluían tareas verbales y de ejecución, y a pesar de que este agrupamiento seguía una estrategia más racional que psicométrica se mantuvo en las primeras revisiones de la escala (WISC, WISC-R, WISC-III). La tercera versión de la escala, WISC-III, introduce en 1991 factores más discretos que miden habilidades cognitivas específicas (Rosas \& Pizarro, 2018), así la escala verbal contenía los factores de "Comprensión Verbal" y "Ausencia de Distractibilidad y la escala ejecutiva, los factores, "Organización Perceptual" y "Velocidad de Procesamiento" (Ramirez \& Rosas, 2007; Wechsler, 2014).

En la versión WISC-IV, del año 2003, basada en los avances de la investigación en evaluación cognitiva y neuropsicológica y el uso de técnicas de análisis factorial, las tradicionales escalas verbal y ejecutiva son reemplazadas por una estructura de cuatro factores que dan cuenta del coeficiente intelectual total. Estos son: "Comprensión Verbal", "Razonamiento Perceptual", "Velocidad de Procesamiento" y un cambio en la denominación del factor "Ausencia de Distractibilidad" (presente en WISC-III) por el de "Memoria de Trabajo", evidenciando una evolución y precisión en la comprensión de dicho constructo cognitivo (Labin, Brenlla, \& Taborda, 2018; Wechsler, 2014). 
A partir de 2006 diversos autores, explorando la estructura factorial de WISC-IV y de la Escala de Inteligencia de Wechsler para adultos (WAISIV) detectaron la emergencia de un modelo alternativo de cinco factores común para ambas escalas, y, a partir del desarrollo de la cuarta versión de la Escala Wechsler de Inteligencia para preescolares (WPPSI-IV) en 2012, las escalas Wechsler comienzan a incluir la actual configuración pentafactorial a la cual se adscribe el WISC-V (Wechsler, 2014). Esta última versión, publicada el año 2014, contempla una estructura factorial compuesta por un coeficiente intelectual total y cinco índices, que mantiene los factores de "Compresión Verbal", "Memoria de Trabajo" y "Velocidad de Procesamiento" presentes en el WISC-IV, pero que divide el factor de "Razonamiento Perceptivo" en "Razonamiento Visoespacial" y "Razonamiento Fluido" (Canivez, Watkins, \& McGill, 2019; Flanagan \& Alfonso, 2017; Forns \& Amador, 2017; Kaufman, Raiford, \& Coalson, 2016; Niileksela \& Reynolds, 2019; Weiss et al., 2019; Wechsler, 2014).

El WISC-V, por otra parte incorpora varias mejoras en relación a su antecesor que incluyen la actualización de sus fundamentos teóricos, aumento de su adecuación y facilidad de uso, y mejoras en sus propiedades psicométricas y utilidad clínica (Wechsler, 2014). La escala WISC-V integra los aportes más recientes del "Modelo Jerárquico de Inteligencia CHC" (Cattel,1963; Cattell-Horn,1978; Carroll, 1993 en Forns \& Amador, 2017), así como también la evidencia emanada de investigaciones en Psicología del Desarrollo, Neurociencia Cognitiva y los modelos de medida de la Memoria de Trabajo. El modelo CHC considera una estructura jerárquica que incluye un factor de inteligencia general, cinco factores primarios amplios o broad level, los cuales a su vez, contienen habilidades cognitivas más específicas (próximas o narrow level), medidas en cada uno de los subtest que conforman la escala (Brenlla, 2013; Kaufman et al., 2016; Scheiber, 2016).

A pesar de que esta estructura de cinco factores ha sido explorada y confirmada en la muestra de estandarización estadounidense (Wechsler, 2014) y en diversos países como España, Canadá y Reino Unido, existen autores que expresan sus aprehensiones en torno a la existencia de cinco factores, luego de realizar revisiones independientes y nuevos análisis que hacen más defendible un modelo factorial compuesto por cuatro índices. Además, critican la retención de un modelo que presenta un coeficiente estandarizado de 1.0 en el path entre inteligencia general y el factor de "Razonamiento Fluido" argumentando que sería empíricamente redundantes. Por otra parte, consideran que la carga cruzada de la subprueba de "Aritmética" en los factores de Comprensión Verbal, Razonamiento Fluido y Memoria de Trabajo, atenta contra la parsimonia del modelo (Beaujean, 2016; Canivez et al., 2016; Canivez et al., 2019; Fenollar-Cortés \& Watkins, 2019). Otros, como Weiss et al., (2019), plantean que a pesar de que aún existe un debate sobre la pertinencia de los modelos de 4 o 5 factores, este último ha mostrado un buen ajuste tanto para muestras normativas como clínicas lo que es suficiente para avalar su utilidad interpretativa. Además en torno al coeficiente estandarizado de 1.0 en el sendero entre inteligencia general y el factor de Razonamiento Fluido lo atribuyen a que este factor sería prácticamente un sinónimo del coeficiente intelectual total. Por último, sobre la carga cruzada de Aritmética, Weiss et al., 2019, plantean que esto no se considera un problema, si no que sólo reafirma el hecho de que el razonamiento matemático explica habilidades cognitivas incorporadas en estos tres índices.

\section{Desarrollo de WISC-V en Chile}

El año 2018 se publicó la versión chilena de WISC-V, previo a un proceso de adaptación y estandarización en base a una muestra normativa de 754 niños, niñas y adolescentes (NNA) (Rosas et al., 2021). Los análisis respecto de la validez estructural de la escala en la muestra chilena, validan el modelo de cinco factores propuesto por el autor para la muestra estadounidense, apoyando la existencia de un modelo jerárquico de cinco factores tanto para las 15 subpruebas que componen la escala (10 subpruebas primarias y 5 complementarias) como para las 10 subpruebas primarias que se incluyen en el nivel de índices principales (Rosas et al., 2021).

Rodríguez-Cancino, et al. (2021) sometieron a comprobación el modelo factorial compuesto por las 15 subpruebas primarias y complementarias de 
WISC-V en una muestra de NNA de procedencia rural en Chile, obteniendo adecuados índices de bondad de ajuste, pero con un valor impropio en el peso factorial de Razonamiento Fluido en el Factor General de inteligencia y una carga cruzada de la subprueba de Aritmética con saturaciones en tres factores, al igual que en los modelos factoriales de otros países (Beaujean, 2016; Canivez et al., 2016; Canivez et al., 2019; Fenollar-Cortés \& Watkins, 2019). Adicionalmente, se obtuvo evidencia de un adecuado ajuste psicométrico para el modelo que incluye las 10 subpruebas primarias y la configuración pentafactorial de WISC-V a nivel de los índices principales, lo que aporta evidencia de la validez basada en la estructura interna del instrumento para la muestra rural chilena.

Van de Vijver, Weiss, Saklofske, Batty y Prifitera (2019) refieren que, dado que la manifestación de las habilidades cognitivas están ampliamente determinadas por factores contextuales y puede diferir a través de las distintas culturas, es necesario realizar investigaciones de invarianza factorial orientadas a demostrar la universalidad del constructo de inteligencia que sustenta la interpretación de las puntuaciones de WISC.

Establecer la invarianza en el WISC-V demostraría que las medidas subyacentes de la inteligencia se fundamentan en la misma estructura teórica para los subgrupos que se están comparando (Pauls, Daseking, \& Peterman, 2019) y que las diferencias en las puntuaciones se asocian a diferencias confiables en las dimensiones intelectuales medidas $y$ no a características propias del grupo de pertenencia, y por tanto, certifican la adecuación de las inferencias que se realizan a partir de las puntuaciones obtenidas por personas de distintos grupos y su posibilidad de comparación (Pauls et al., 2019; Pedrero, 2019; Van de Schoot, Lugtig, \& Hox, 2012).

\section{Invarianza factorial del WISC-V}

El primer estudio que examinó la invarianza factorial de WISC-V fue conducido por Chen et al. (2015) quienes la exploraron a través del género en la muestra normativa estadounidense (2200 participantes). Sus resultados alcanzaron el nivel de invarianza factorial más alto (estricta), demostrando que las subpruebas que componen la escala presentan los mismos constructos teóricos latentes subyacentes, la misma intensidad de las relaciones entre las subpruebas y los factores, la misma validez de cada factor de primer orden y las mismas comunalidades, independientemente del género de los participantes. Entre sus resultados destacan que tanto en niños como en niñas se aprecia la presencia de la carga cruzada de "Aritmética" en los factores de "Razonamiento Fluido", "Memoria de Trabajo" y "Comprensión Verbal", argumentando que el razonamiento matemático está compuesto por las habilidades de estos tres factores. También se refieren a la carga estandarizada de 1.00 del índice de Razonamiento Fluido en el factor de orden superior, lo cual interpretan como una demostración de que "el razonamiento fluido es una piedra angular de la cognición humana" (p. 4). Los autores concluyen que de acuerdo con los hallazgos referidos a la invarianza factorial es posible sustentar el mismo enfoque interpretativo y comparaciones significativas de WISC-V entre niños y niñas.

Por otra parte, Scheiber (2016) examinó la invarianza factorial de WISC-V a través de etnia y género, en una muestra de 2637 niños y niñas afroamericanos, hispanos y caucásicos en Estados Unidos. Los resultados demostraron la invarianza configural, métrica y escalar para los 6 grupos en los que fue explorada, lo que para esta autora implica que la estructura factorial es consistente entre los grupos, tanto por etnia como por género, y por ende las inferencias extraídas de las puntuaciones son igualmente significativas para niños y niñas hispanos, afroamericanos $\mathrm{y}$ caucásicos. La autora concluye que, a nivel de implicancias clínicas, este estudio otorga evidencia empírica adicional para el uso del WISC-V en los grupos de niños y niñas pertenecientes a las etnias estudiadas.

Reynolds y Keith (2017), por su parte, en un estudio de invarianza factorial a través de los rangos etarios en la muestra de estandarización estadounidense de WISC-V obtuvieron evidencia de invarianza estricta, que permite determinar la presencia de la misma estructura factorial y los mismos constructos subyacentes, independientemente de la edad de los participantes. Van de Vijver et al., (2019) examinaron la invarianza del modelo de cinco 
factores, en distintos países en los que había sido adaptada la escala (Australia, Nueva Zelanda, Francia, Alemania, Reino Unido, Estados Unidos, entre otros). Para este análisis utilizaron los puntajes estandarizados de las 10 subpruebas primarias. Sus resultados evidencian soporte al nivel de invarianza configural y métrica y más débilmente, a la invarianza escalar. De acuerdo con estos autores, estos resultados permitirían sostener la existencia de significados psicológicos compartidos para las subpruebas de WISC-V en los países estudiados, sustentando la aplicabilidad global de la estructura factorial jerárquica de la escala.

Pauls et al., (2019) realizaron un análisis de invarianza de medida a través del género, en la versión alemana de WISC-V. Sus resultados apoyan la equivalencia de 11 de las 15 subpruebas, sustentando la posibilidad de interpretar las puntuaciones en el test de la misma forma para hombres y mujeres, considerando el nivel de invarianza escalar parcial alcanzado. Los autores de este estudio alertan sobre las dificultades observadas en relación a la carga factorial de la subprueba de "Matrices de Razonamiento" sobre el índice de "Razonamiento Fluido", y la carga cruzada de la subprueba de Aritmética presente en el modelo propuesto por Wechsler (2014). Además, resaltan que la evidencia de estudios recientes coloca en duda la existencia de 5 factores al encontrar que un modelo bifactorial de 4 factores presenta un mejor ajuste para la estructura factorial actualmente hipotetizada para esta versión de WISC. En la misma línea, Smith y Graves (2020) exploraron la invarianza según el género de los participantes en una muestra de 647 NNA afroamericanos, alcanzando el nivel de invarianza métrica, en la que dos subpruebas que variaron entre niños y niñas indican que los puntajes de dos índices factoriales no pueden interpretarse al mismo nivel. Por su parte, Chen et al. (2020) encontraron un nivel de invarianza factorial completa, según edad y género, en una muestra de 1.034 NNA de Taiwan apoyando el enfoque interpretativo de la escala para esta muestra.

De acuerdo con los antecedentes hasta aquí expuestos la pregunta de investigación que guía el presente estudio es: ¿Es la estructura factorial del WISC-V invariante según la procedencia (rural- urbana) de niños, niñas y adolescentes chilenos?. Esto es altamente relevante dado que actualmente no existen en Chile estudios que hayan explorado este aspecto, y se aproxima el uso masivo de la nueva versión de la escala a nivel nacional (Ordenanza $\mathrm{N}^{\circ} 05$ de la División General de Educación, del 28 de agosto de 2019).

Además, cabe destacar que en Chile de acuerdo al Decreto 170 del Ministerio de Educación (2009) se exige el uso de esta escala como requisito para el ingreso de los NNA a Proyectos de Integración Escolar y actualmente se continúa utilizando la versión previa (WISC-III de 2007) en ausencia de estudios de su funcionamiento psicométrico en subgrupos de la población. Dado que los lineamientos éticos contenidos en las directrices internacionales sobre el adecuado uso de test (ITC, 2013; AERA, APA, \& NCME, 2014) exigen el estudio de subgrupos, esta investigación se vuelve indispensable. Por esto, se pretende aportar de manera pionera en Chile a requerimientos contenidos en los marcos éticos nacionales e internacionales, así como también al ámbito profesional de los psicólogos usuarios de WISC-V, proveyendo de información psicométrica que oriente el adecuado uso de la escala con niños de procedencia rural.

En concreto, siguiendo los lineamientos contenidos en los "Estándares para Pruebas Educativas y Psicológicas" (AERA, APA, \& NCME, 2014) el presente estudio se enmarca en una línea de investigación que explora la imparcialidad de los test de uso masivo, como un aspecto fundamental de la validez, indagando si los constructos cognitivos medidos y contenidos en la estructura factorial de WISC-V son similares y no varían en función de la procedencia urbana o rural de los evaluados. Por tanto, se busca evaluar si la estructura pentafactorial de segundo orden de la escala WISC-V es psicométricamente equivalente en estos dos grupos considerando que demostrar la invarianza de la medida robustece el examen de la validez basada en estructura interna de un test y suele ser un indicador de su imparcialidad (Elosua, 2003; Pauls et al., 2019; Pedrero, 2019)

La relevancia de este estudio radica en que al ser de carácter pionero en Chile, se constituye no sólo en un aporte a nivel teórico en la evaluación de la inteligencia, si no que también contribuye a 
Tabla 1. Distribución de la Muestra Urbana y Rural

\begin{tabular}{|c|c|c|c|c|c|c|c|c|c|c|}
\hline \multirow{3}{*}{$\frac{\text { Dependencia }}{\text { Edad }}$} & \multicolumn{6}{|c|}{ Grupo Urbano } & \multirow{3}{*}{$\%$} & \multirow{2}{*}{\multicolumn{2}{|c|}{ Grupo Rural }} & \multirow{3}{*}{$\%$} \\
\hline & \multicolumn{2}{|c|}{ Municipal } & \multicolumn{2}{|c|}{ Part. Subvenc. } & \multicolumn{2}{|c|}{ Privada } & & & & \\
\hline & Hombre & Mujer & Hombre & Mujer & Hombre & Mujer & & Hombre & Mujer & \\
\hline 6 & 6 & 7 & 7 & 9 & 2 & 1 & 10 & 7 & 9 & 10 \\
\hline 7 & 6 & 7 & 8 & 10 & 1 & 2 & 11 & 8 & 9 & 11 \\
\hline 8 & 4 & 4 & 7 & 7 & 1 & 1 & 7 & 6 & 6 & 08 \\
\hline 9 & 5 & 4 & 8 & 7 & 1 & 1 & 8 & 7 & 6 & 08 \\
\hline 10 & 4 & 6 & 6 & 9 & 1 & 1 & 8 & 5 & 8 & 08 \\
\hline 11 & 5 & 6 & 8 & 9 & 1 & 1 & 9 & 7 & 8 & 09 \\
\hline 12 & 7 & 5 & 9 & 8 & 1 & 1 & 10 & 9 & 7 & 10 \\
\hline 13 & 5 & 4 & 8 & 7 & 1 & 1 & 8 & 7 & 6 & 07 \\
\hline 14 & 4 & 7 & 7 & 10 & 1 & 2 & 10 & 6 & 10 & 10 \\
\hline 15 & 6 & 7 & 9 & 10 & 1 & 1 & 11 & 8 & 9 & 11 \\
\hline 16 & 6 & 4 & 7 & 6 & 1 & 1 & 8 & 7 & 5 & 08 \\
\hline Total & 58 & 61 & 84 & 92 & 12 & 13 & 100 & 77 & 83 & 100 \\
\hline
\end{tabular}

la realización de una medición más justa y que releva la importancia de explorar la pertinencia cultural de los test que se utilizan para determinaciones de apoyo que impactan la trayectoria vital y escolar de los NNA de un subgrupo de la población, como lo es la procedencia rural.

Considerando las etapas en el proceso de verificación de la invarianza (Putnick \& Bornstein, 2016; Vandenberg \& Lance, 2000), las hipótesis que se sometieron a prueba para evaluar la equivalencia urbano-rural del modelo jerárquico pentafactorial del WISC-V, fueron las siguientes:

H1) La estructura factorial (número de factores y número de indicadores) de WISC-V es equivalente en las muestras urbana y rural (Invarianza Configural);

$\mathrm{H}_{0}$ : La estructura factorial no es equivalente entre las muestras

$\mathrm{H}_{1}$ : La estructura factorial es equivalente entre las muestras

H2) Las cargas factoriales de las subpruebas del WISC-V son equivalentes en las muestras urbana y rural (Invarianza Métrica);

$\mathrm{H}_{0}$ : Las cargas factoriales no son equivalentes entre las muestras

$\mathrm{H}_{1}$ : Las cargas factoriales son equivalentes entre las muestras

H3) Los interceptos de las subpruebas del WISC-V son equivalentes en las muestras urbana y rural (Invarianza Escalar);

$\mathrm{H}_{0}$ : Los interceptos no son equivalentes entre las muestras

$\mathrm{H}_{1}$ : Los interceptos son equivalentes entre las muestras
H4) Las varianzas residuales de las subpruebas del WISC-V son equivalentes en las muestras urbana y rural (Invarianza Residual).

$\mathrm{H}_{0}$ : Las varianzas residuales no son equivalentes entre las muestras

$\mathrm{H}_{1}$ : Las varianzas residuales son equivalentes entre las muestras

\section{Método}

\section{Participantes}

La muestra de procedencia urbana corresponde a datos secundarios obtenidos del Proyecto de estandarización de la Escala WISC-V en Chile, por parte del equipo de investigación del Centro de Desarrollo de Tecnologías de Inclusión de la Pontificia Universidad Católica de Chile (CEDETI-UC). En esta muestra se incluyen 693 NNA provenientes de 5 zonas urbanas del país, seleccionados a través de un muestreo intencionado no probabilístico. Los criterios de inclusión fueron: a) Edad entre 6 años y 16 años, 11 meses, b) Procedencia urbana y c) Ausencia de diagnóstico de necesidades educativas especiales permanentes o transitorias.

De esta muestra se seleccionaron 320 escolares, a través de un muestreo aleatorio estratificado (Ver Tabla 1). Dado que en Chile los establecimientos educativos se organizan en torno a tres tipos de dependencia (municipal, particular subvencionada y privada), a fin de aproximarse a una mayor representación de la realidad nacional, el muestreo utilizado consideró este criterio (Ministerio de Educación, 2018) incluyendo una cantidad de casos proporcional a la frecuencia de la matrícula de niños por cada tipo de dependencia 
en el país (Municipal=36\%, Particular subvencionada $=55 \%$, Privada $=8 \%$ ).

Por su parte, la muestra de procedencia rural estuvo conformada por 160 NNA seleccionados a través de un muestreo intencionado, no probabilístico. Los criterios de inclusión fueron: (a) Edad entre 6 años y 16 años, 11 meses, (b) Procedencia rural, y (c) Ausencia de diagnóstico de necesidades educativas especiales permanentes o transitorias, de acuerdo al reporte de directivos y profesores de su establecimiento educacional. Para este estudio, se consideró como ruralidad todo asentamiento humano cuya población económicamente activa se desempeña en sectores como la ganadería, artesanía, agricultura, pesca, minería, entre otros, y cuya población es menor a 2.000 habitantes dispersos o agrupados en diversas villas (Pérez, 2001; Instituto Nacional de Estadísticas [INE], 2004).

\section{Instrumento}

La escala Wechsler de Inteligencia para niños, quinta edición, estandarización chilena (WISC-V) es un instrumento clínico que mide habilidades intelectuales a través de 15 subpruebas, en NNA desde los 6 a 16 años de edad. Los análisis sobre sus propiedades psicométricas reportados por Rosas y Pizarro (2018) y Rosas et al. (2021), muestran adecuados niveles de confiabilidad y evidencias de validez factorial. Específicamente en población rural, el estudio de Rodríguez-Cancino et al. (2020) previamente citado demostró apropiados índices de ajuste para la configuración pentafactorial de WISC-V a nivel de los índices principales, aportando evidencia de la validez basada en la estructura interna de la escala.

La versión chilena de esta escala incluye 15 subpruebas, cuyas puntuaciones estandarizadas se expresan en puntaje escala $(M=10, D S=03)$ y cinco índices principales, que permiten obtener puntajes compuestos $(M=100, D S=15)$.

De acuerdo con Rosas y Pizarro (2018) las subpruebas que componen WISC-V para Chile se dividen en 10 subpruebas primarias $\mathrm{y}$ cinco complementarias, que se distribuyen en los cinco dominios cognitivos, de la siguiente forma:
Comprensión Verbal $(\mathrm{CV})$, incluye las subpruebas de Analogías $\left(\mathrm{AN}^{1}\right)$, Vocabulario $\left(\mathrm{VO}^{1}\right)$, Información $\left(\mathrm{IN}^{2}\right)$ y Comprensión $\left(\mathrm{COM}^{2}\right)$;

Razonamiento Visoespacial (VE), conformado por las subpruebas de Construcción con Cubos $\left(\mathrm{CC}^{1}\right)$ y Rompecabezas Visuales $\left(\mathrm{RV}^{1}\right)$;

Razonamiento Fluido (RF), que contiene las subpruebas de Matrices de Razonamiento $\left(\mathrm{MR}^{1}\right)$, Balanzas $\left(\mathrm{BA}^{1}\right)$ y Aritmética $\left(\mathrm{AR}^{2}\right)$;

Memoria de Trabajo (MT), que incluye las subpruebas de Retención de Dígitos $\left(\mathrm{RD}^{1}\right)$, Retención de Imágenes $\left(\mathrm{RI}^{1}\right)$ y Secuenciación de Letras y Números $\left(\mathrm{SLN}^{2}\right)$; y

Velocidad de Procesamiento (VP) constituido por las subpruebas de Claves $\left(\mathrm{CLA}^{1}\right)$, Búsqueda de Símbolos $\left(\mathrm{BS}^{1}\right)$ y Cancelación $\left(\mathrm{CAN}^{2}\right)$.

WISC-V contempla la presencia de al menos tres niveles de análisis para la interpretación de sus puntuaciones. El primero, a nivel de escala total, es el resultado de las puntuaciones escalares de siete subpruebas primarias que permiten dar cuenta del Coeficiente Intelectual Total. Un segundo nivel corresponde al de los Índices Principales, obtenidos a partir de la aplicación de 10 subpruebas primarias. El tercer nivel, introduce cinco índices adicionales, que permiten enriquecer los resultados explorando habilidades mas específicas (Forns \& Amador, 2017; Kaufman et al., 2016; Rosas \& Pizarro, 2018; Rosas et al., 2021; Weiss et al., 2019)

Los análisis de consistencia interna evidencian que no existen diferencias estadísticamente significativas entre los coeficientes de confiabilidad $(p=.845)$ para la muestra urbana $(\alpha$.797) y para la muestra rural $(\alpha=.803)$.

\section{Procedimiento}

Para la recolección de los datos inicialmente se contactaron establecimientos educacionales, se les informó de los objetivos, procedimiento y consideraciones éticas de este estudio y se enviaron cartas de invitación a los padres de los niños, niñas y adolescentes que cumplían los criterios de inclusión y que fueron previamente seleccionados. Junto con esta carta se envió el

\footnotetext{
${ }^{1}$ Subprueba Primaria

${ }^{2}$ Subprueba Complementaria
} 
documento de Consentimiento Informado que incluía los objetivos del estudio, los resguardos éticos y las condiciones de administración de los instrumentos (por ejemplo, que serían aplicados por evaluadores calificados, en dependencias de los establecimientos educacionales y en horario regular de clases). Una vez que los padres autorizaron la participación de sus hijos e hijas, se citó a los niños, niñas y adolescentes seleccionados, se les explicaron los objetivos y condiciones de la investigación $\mathrm{y}$, previa aceptación de los escolares expresada en el documento de Asentimiento Informado, se procedió a la aplicación de los instrumentos.

Todos los participantes de este estudio fueron evaluados en forma individual por examinadores previamente entrenados, en sesiones de trabajo de 1 hora y 45 minutos aproximadamente, y en la medida de lo posible, en un solo día. Cuando esto no fue posible la aplicación de instrumentos se realizó en dos sesiones, dentro de una misma semana de clases.

Con respecto a los resguardos éticos, cabe destacar que este estudio se adscribe a los principios rectores de la American Psychological Association (APA, 2010) para la investigación con seres humanos y el correspondiente protocolo de investigación fue aprobado por el Comité Ético Científico de la Universidad de La Frontera en Temuco, Chile. Los documentos de Consentimiento y Asentimiento Informado, explicitaron a los participantes y sus padres los objetivos del estudio, las condiciones de administración de la escala, y el derecho a retirarse de la investigación, en cualquier momento, sin tener que entregar ningún tipo de explicación y sin que ello significara ningún perjuicio. Adicionalmente, se explicitó el procedimiento de manejo de información y resguardo de su confidencialidad, señalando que sólo sería utilizada con fines científicos y académicos. Estos documentos incluyeron los datos de contacto de la investigadora responsable, en el caso de que el/la participante o sus padres requirieran mayor información. Finalmente cabe agregar que se ofreció a los establecimientos educacionales y a las familias de los participantes, una síntesis de los resultados a modo de retribución por ser parte de esta investigación.

\section{Análisis de datos}

El modelo pentafactorial escogido para realizar el análisis de invarianza fue el de 10 subpruebas primarias propuesto por Wechsler (2014) para la muestra de estandarización estadounidense y replicado en la muestra de estandarización chilena (Rosas et al., 2021), dado que en la investigación previamente realizada por Rodríguez-Cancino et al. (2021), en la muestra rural éste presentó una mejor solución factorial que el modelo de 15 subpruebas.

Siguiendo las indicaciones de Dimitrov (2010) para examinar la invarianza factorial en modelos de segundo orden, como paso preliminar al análisis de invarianza factorial, se testeó este modelo pentafactorial, por separado para el grupo de escolares de procedencia urbana y para los de procedencia rural.

Luego de esto se testeó un modelo factorial para la totalidad de la muestra $(n=480)$ que se estableció como modelo de línea base, el cual está especificado en la Figura 1. En este modelo las 10 subpruebas primarias de WISC-V (AN, VO, CC, $\mathrm{RV}, \mathrm{MR}, \mathrm{BA}, \mathrm{RD}, \mathrm{RI}, \mathrm{CLA}, \mathrm{BS})$ se agrupan en 5 factores de primer orden (CV, VE, RF, MT, VP), los cuales dan cuenta de un indicador de inteligencia general de segundo orden.

A través de la técnica de Análisis Factorial Confirmatorio de Multigrupo (AFC-MG) se probaron distintos niveles de modelos anidados que incluían sucesivamente más restricciones en cada nivel, de manera simultánea para ambos grupos (Dimitrov, 2010; Putnick \& Bornstein, 2016), de la siguiente forma:

a) Invarianza Configural, examinando si la estructura factorial es equivalente para ambos grupos, sin restricciones de invarianza

b) Invarianza Métrica, testeando si las cargas factoriales son equivalentes entre los grupos, restringiéndolas y luego comparando su ajuste con el nivel de invarianza configural.

c) Invarianza Escalar, confirmando si las medias de los indicadores son equivalentes entre los grupos, por lo que se aplican restricciones a los interceptos y se compara su ajuste con el nivel de invarianza métrica.

d) Invarianza Residual, evaluando si los residuos de los indicadores son equivalentes entre los grupos, restringiéndolos y luego comparando su ajuste con el nivel de invarianza escalar. 


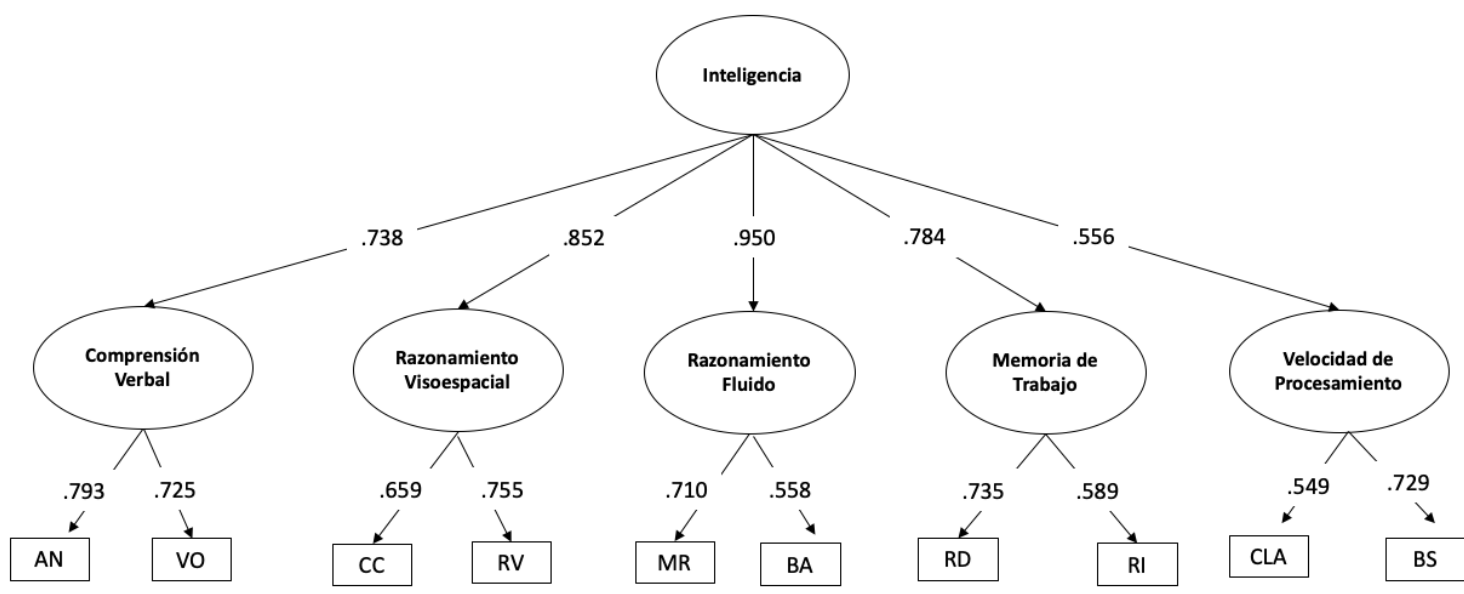

Figura 1. Modelo Pentafactorial de WISC-V como Línea Base obtenido con la muestra total $(\mathrm{n}=480)$ Nota. AN=Analogías; $\mathrm{VO}=$ Vocabulario; $\mathrm{CC}=$ Construcción con Cubos; RV=Rompecabezas Visuales; MR=Matrices de Razonamiento;

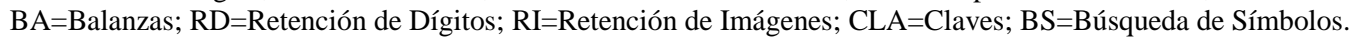

Tabla 2. Índices de ajuste para el modelo pentafactorial de segunto orden del WISC-V para los grupos urbano y rural, por separado

\begin{tabular}{lcccccccc}
\hline & \multicolumn{7}{c}{ Índices de ajuste del modelo } \\
\cline { 2 - 9 } Grupo & $\chi^{2}$ & $g l$ & $p$ & $C F I$ & TLI & SRMR & RMSEA & $90 \%$ IC \\
\hline Urbano & 28,174 & 30 & .5612 & 1,000 & 1,004 & 0,025 & 0,000 & {$[, 000,039]$} \\
Rural & 29,455 & 30 & .4938 & 1,000 & 1,002 & 0,035 & 0,000 & {$[, 000,058]$} \\
\hline
\end{tabular}

Nota. $\chi^{2}=$ Chi-cuadrado; $g l=$ grados de libertad; $C F I=I ́$ ndice de Ajuste Comparativo; $T L I=I ́ n d i c e$ Tucker-Lewis; SRMR=Raíz Cuadrada Media Residual Estandarizada; RMSEA=Raíz Cuadrada Media del Error de Aproximación.

Cabe destacar que en este tipo de análisis sólo una vez que se ha establecido un nivel de invarianza previo, es posible testear el nivel siguiente.

Una vez demostrada la normalidad multivariada mediante el Test de Mardia se usó el estimador Máxima Verosimilitud (ML, por su sigla en inglés), tanto para los AFC como para los AFC-MG, realizados en este estudio. Para evaluar el ajuste de los modelos factoriales se consideró la relación $\chi^{2} / \mathrm{gl}$, el Error Cuadrático Medio de Aproximación (RMSEA), el Índice de Ajuste Comparativo (CFI), y el Índice de Tucker-Lewis (TLI). De acuerdo con esto, un ajuste óptimo consideró CFI y TLI $\geq .95$, y RMSEA $<.05$ y un ajuste razonable, CFI y TLI $\geq .90$, RMSEA $<.08$ y una relación $\chi^{2} / \mathrm{gl}$ menor a 2 (Hu \& Bentler, 1999; Tabachnick \& Fidell, 2014). El valor de .95 se estableció como punto de corte para determinar un ajuste aceptable de todos los índices que van desde 0 a 1, donde 1 representa un ajuste perfecto.

A fin de determinar la invarianza en la comparación de modelos (métrica versus configural, escalar versus métrica) se tomaron las recomendaciones de Chen (2007) quien plantea que las variaciones $\geq .010$ en $\mathrm{CFI}, \geq .015$ en RMSEA o $\geq .030$ en SRMR indicarían ausencia de invarianza métrica. Además, a nivel de invarianza escalar y estricta una diferencia $\geq .010$ en CFI, $\geq .015$ en RMSEA o $\geq .010$ en SRMR sugerirían ausencia de invarianza. Cabe destacar que estos mismos criterios se consideraron en estudios previos de invarianza factorial para WISC-V en otros países (Chen et al., 2015; Pauls et al., 2019; Reynolds \& Keith, 2017; Scheiber, 2016).

Los datos fueron analizados con los softwares IBM SPSS Statistics 22 y con Mplus 7.11. Todos los análisis fueron realizados con los puntajes escala de las 10 subpruebas primarias de WISC-V.

\section{Resultados}

El modelo factorial de segundo orden sin restricciones (línea base) se evaluó con la muestra total y por separado para la muestra rural y urbana.

Los resultados del AFC para la muestra completa, mostraron adecuados índices de ajuste, $\chi_{(28,807)}{ } / g l_{(30)}=0,960 ; R M S E A=0,000$ (IC 90\% [0,000 0,033]); CFI=1,000; TLI=1,002; $S R M R=0,021$, y evidencian que la estructura factorial propuesta por Wechsler (2014), representa apropiadamente un modelo factorial que contempla una muestra de escolares de 
Tabla 3. Índices de Ajuste del AFC Multigrupo de segundo orden del WISC-V y comparación de los Modelos de Invarianza

\begin{tabular}{|c|c|c|c|c|c|c|c|c|c|c|c|c|c|c|}
\hline \multirow{2}{*}{$\begin{array}{l}\text { Modelo de } \\
\text { Invarianza }\end{array}$} & \multicolumn{9}{|c|}{ Índices de ajuste } & \multicolumn{5}{|c|}{ Comparación de Modelos } \\
\hline & $\chi^{2}$ & gl & $p$ & $\chi^{2} / g 1$ & CFI & TLI & $\begin{array}{l}\text { RMSEA } \\
\text { [IC 90\%] }\end{array}$ & SRMR & AIC & Comparación & $\Delta \chi^{2}$ & $\Delta \mathrm{df}$ & $\Delta \mathrm{CFI}$ & $\triangle \mathrm{RMSEA}$ \\
\hline $\mathrm{M}_{0}$ : Configural & 72,806 & 67 & .293 & 1,087 & 0,994 & 0,993 & $\begin{array}{c}0,019 \\
{[0,0000,049]}\end{array}$ & 0,045 & $22.435,305$ & - & - & - & - & - \\
\hline $\begin{array}{l}\mathrm{M}_{1}: \text { Métrica } \\
\left(1^{\circ} \text { Orden }\right)\end{array}$ & 96,272 & 75 & .049 & 1,284 & 0,980 & 0,976 & $\begin{array}{c}0,034 \\
{[0,0020,053]}\end{array}$ & 0,051 & $22.442,771$ & $\mathrm{M}_{1}$ vs $\mathrm{M}_{0}$ & 23,466 & 8 & 0,014 & 0,015 \\
\hline $\begin{array}{l}\mathrm{M}_{1 \mathrm{a}} \text { : Métrica Parcial } \\
\left(1^{\circ} \text { Orden }\right)\end{array}$ & 88,483 & 74 & .120 & 1,196 & 0,986 & 0,983 & $\begin{array}{c}0,029 \\
{[0,0000,049]}\end{array}$ & 0,049 & $22.436,982$ & $\mathrm{M}_{1 \mathrm{a}}$ vs $\mathrm{M}_{0}$ & 15,677 & 7 & 0,008 & 0,005 \\
\hline $\begin{array}{l}\mathrm{M}_{2}: \text { Métrica } \\
\left(2^{\circ} \text { Orden }\right)\end{array}$ & 89,345 & 78 & .179 & 1,145 & 0,989 & 0,987 & $\begin{array}{c}0,025 \\
{[0.0000 .046]}\end{array}$ & 0,051 & $22.429,843$ & $\mathrm{M}_{2}$ vs $\mathrm{M}_{1 \mathrm{a}}$ & 0,862 & 4 & 0,004 & 0,004 \\
\hline $\begin{array}{l}\mathrm{M}_{3}: \text { Escalar } \\
\text { (Indicadores) }\end{array}$ & 152,382 & 88 & $<.001$ & 1,731 & 0,938 & 0,937 & $\begin{array}{c}0,055 \\
{[0.0400 .070]}\end{array}$ & 0,081 & $22.472,880$ & $\mathrm{M}_{3}$ vs $\mathrm{M}_{2}$ & 63,037 & 10 & 0,051 & 0,03 \\
\hline $\begin{array}{l}\mathrm{M}_{3 \mathrm{a}}: \text { Escalar parcial } \\
\text { (Indicadores) }\end{array}$ & 133,711 & 86 & .001 & 1,537 & 0,958 & 0,956 & 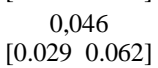 & 0,072 & $22.454,120$ & $\mathrm{M}_{3 \mathrm{a}}$ vs $\mathrm{M}_{2}$ & 44,366 & 8 & 0,031 & 0,021 \\
\hline
\end{tabular}

Nota 1. M0=modelo configural (sin restricciones de invarianza); M1=modelo de invarianza métrica de primer orden (igualdad de cargas en los factores de primer orden); M1a=modelo de invarianza métrica parcial de primer orden (igualdad de cargas en los factores de primer orden, salvo en la subprueba de Analogías); M2=M1a+modelo de invarianza métrica de segundo orden (igualdad de cargas en el factor de segundo orden); M3=M2 + modelo de invarianza escalar (igualdad de interceptos de los indicadores); M3a=modelo de invarianza escalar parcial a nivel de indicadores (igualdad de interceptos de los indicadores, salvo en las subpruebas de Analogías y Búsqueda de Símbolos).

Nota 2: $\chi^{2}=$ Chi-cuadrado; $g l=$ grados de libertad; $C F I=$ Índice de Ajuste Comparativo; $T L I=$ Índice Tucker-Lewis; $R M S E A=$ Raíz Cuadrada Media del Error de Aproximación; $S R M R=$ Raíz Cuadrada Media Residual Estandarizada; $A I C=$ Criterio de Información de Akaike; $\Delta \chi^{2}=$ diferencia en Chi-cuadrado entre los modelos comparados; $\Delta g l=$ diferencia en grados de libertad entre los modelos comparados; $\triangle C F I=$ diferencia en CFI entre los modelos comparados. Las diferencias en la comparación de modelos $(\Delta)$ representan valores absolutos.

procedencia urbana y rural de Chile (ver Figura 1).

Los resultados del AFC para los grupos urbano y rural, por separado, también muestran índices de ajustes adecuados (ver Tabla 2).

Siguiendo la recomendación de Dimitrov (2010), se realizó análisis de invarianza multigrupo de segundo orden. El nivel de invarianza configural $\left(\mathrm{M}_{0}\right)$ fue examinado sobre la base de la estimación de un modelo para ambos grupos simultáneamente, sin restricciones de invarianza. Tal como se observa en la Tabla 3, los estadísticos generales de ajuste sugieren que se puede asumir razonablemente un nivel de invarianza configural indicando que la estructura factorial (número de factores y número de indicadores por factor) es equivalente para ambos grupos, es decir, en el modelo se observa que las mismas subpruebas se asocian a los mismos factores latentes de primer orden, y que éstos dan cuenta de la misma forma. Esto implica, un factor de segundo orden y cinco factores de primer orden (con dos indicadores cada uno), tanto para el grupo urbano, como para el rural. Este resultado permitió demostrar la $\mathrm{H} 1$ de este estudio.

Dado que se estableció el nivel de invarianza configural, el siguiente paso consistió en agregar restricciones a las cargas factoriales del modelo a fin de comprobar el nivel de invarianza métrica. Los resultados a nivel de los factores de primer orden $\left(\mathrm{M}_{1}\right)$ muestran adecuados índices de ajuste, sin embargo la variación de CFI obtenida de la comparación entre $\mathrm{M}_{1}$ y $\mathrm{M}_{0}$, evidencia que existen diferencias que sobrepasan el límite admitido en las cargas factoriales según la procedencia urbana o rural. De acuerdo con esto, se rechaza la Hipótesis 2 de invarianza métrica completa y se especifica un modelo de invarianza métrica parcial.

Para esto se decidió realizar un procedimiento para identificar la o las subpruebas variantes, a través de la comparación entre los dos grupos, de las cargas factoriales de todas las subpruebas. Las diferencias entre los pesos $(\lambda)$ indicaron que el desajuste podía atribuirse a la subprueba Analogías $\left(\lambda_{\text {urbano }}=.804, \lambda_{\text {rural }}=.603\right)$, dado que presentó la diferencia más alta en relación a las otras subpruebas comparadas. Una vez identificada, la carga factorial de esta subprueba se dejó fluctuar libremente y se volvió a estimar el modelo. Este paso mejoró sustancialmente el nuevo modelo $\left(\mathrm{M}_{\mathrm{la}}\right)$ evidenciando adecuados índices de ajuste, que al ser comparado con el modelo configural $\left(\mathrm{M}_{0}\right)$ presenta una variación de CFI y RMSEA que permiten aceptar la invarianza métrica parcial en el primer nivel del modelo.

A nivel de factor de segundo orden $\left(\mathrm{M}_{2}\right)$, igualmente existen adecuados índices de ajuste, y la variación de CFI y RMSEA obtenida de la comparación entre $\mathrm{M}_{2}$ y $\mathrm{M}_{1 \mathrm{a}}$, demuestra que no existen diferencias sustanciales entre las cargas factoriales de los grupos comparados. A partir de estos resultados, es posible establecer un nivel de invarianza métrica de tipo parcial $(\mathrm{H} 2)$. 
A continuación, se realizó el análisis del nivel de invarianza escalar $\left(\mathrm{M}_{3}\right)$, para lo cual los interceptos de los indicadores se limitaron a ser iguales en los dos grupos. Los valores de CFI y RMSEA certificaron un buen nivel de ajuste del modelo, sin embargo, la variación de CFI obtenida de la comparación entre $M_{3}$ y $M_{2}$ no otorgan soporte a este nivel de invarianza impidiendo demostrar la hipótesis 3 de este estudio. Una vez identificado que los interceptos de las subpruebas Analogías y Búsqueda de Símbolos varían entre los grupos comparados, se liberaron esos parámetros y se re-estimó el modelo $\left(\mathrm{M}_{3 \mathrm{a}}\right)$, sin que se lograra demostrar la invarianza escalar parcial, por lo que la evaluación de invarianza de medida se detuvo en este paso. Dado que este nivel de invarianza escalar no fue alcanzado, el nivel de invarianza de los residuos no fue testeado, y en consecuencia no se cumple la $\mathrm{H} 4$ de este estudio.

\section{Discusión}

Es relevante mencionar que este es el primer estudio en Chile que proporciona evidencia de la equivalencia factorial, según procedencia urbana o rural, de la escala WISC-V, la cual, como se ha mencionado previamente, es la prueba de inteligencia más utilizada para evaluar el funcionamiento cognitivo de NNA, determinando diagnósticos que permitirán o restringirán el acceso de los escolares a diversas fuentes se apoyo educativo.

Considerando los criterios estadísticos establecidos para determinar la prueba de la hipótesis de invarianza (variaciones de CFI y RMSEA) los resultados obtenidos en esta investigación permiten establecer un nivel de invarianza configural y métrica parcial. A nivel de invarianza configural los hallazgos muestran que la estructura factorial de WISC-V es equivalente para ambos grupos. Este nivel de invarianza, fue igualmente alcanzado en otros estudios que consideraron diversas variables socioestructurales, especialmente de género (Chen et al., 2015; Pauls et al., 2019; Reynolds \& Keith, 2017; Scheiber, 2016), y dan soporte a la posibilidad de considerar que la inteligencia, como constructo general, se estructura de la misma manera para ambos grupos y coincide con la propuesta pentafactorial jerárquica que sostiene Wechsler (2014).
En otras palabras, sería posible afirmar que en el funcionamiento cognitivo de los escolares de procedencia urbana y rural, se aprecia una configuración de las habilidades similar a la estructura jerárquica propuesta por el modelo CHC. Esto significa que las habilidades más específicas (10 subpruebas primarias) se organizan en torno a cinco habilidades más amplias (índices principales o factores de primer orden), que en su conjunto dan cuenta de un constructo de inteligencia o factor de segundo orden (Brenlla, 2013; Kaufman et al., 2016; Scheiber, 2016).

Cabe destacar que si bien este nivel de invarianza sugiere que existe igual relación entre las subpruebas y el constructo medido entre los grupos comparados, esto aún no permite establecer comparaciones del desempeño en las subpruebas entre evaluados urbanos y rurales (Treviño, Sandoval-Hernández, Miranda, Rutkowski, \& Matta, 2019).

En un siguiente paso, sin embargo, los resultados permitieron aceptar un nivel de invarianza métrica parcial, evidenciando que la relación entre los ítems y las variables latentes es equivalente para ambos grupos en la totalidad de los factores de segundo orden y en 9 de las 10 subpruebas primarias a excepción de la subprueba de Analogías. De acuerdo con Putnick y Bornstein (2016) alcanzar el nivel de invarianza métrica significa que cada ítem contribuye al factor latente de una manera similar, por lo que los resultados de este estudio darían cuenta de que en los niños de procedencia rural la subprueba de Analogías, no contribuye al factor de Comprensión Verbal con la misma intensidad que lo hace para los niños urbanos.

Sin desconocer que la evidencia nacional e internacional ha mostrado desempeños más disminuidos en niños procedentes de sectores rurales o vulnerables socioeconómicamente en las tareas que requieren de la habilidad de resolución de problemas a través del uso del lenguaje y el nivel de abstracción verbal, cuando son comparados con sus pares urbanos (Cianci, Orsini, Helbert, \& Pezutti, 2013; Coronel et al., 2006; Eilertsen et al., 2016; Fuica et al., 2014; Labin, Taborda, \& Brenlla, 2015; Morris, Kelsey, \& Martin, 1980; Rodríguez, Rosas, \& Pizarro, 2019), los resultados de este estudio podrían 
sugerir que las diferencias en el nivel de acceso cultural o el nivel educativo de los padres de niños urbanos en comparación a los rurales, favorecería el desempeño de los primeros en tareas que requieren razonamiento verbal medidas en la subprueba de Analogías de WISC-V. Alternativamente, podría indicar diferencias en el proceso de abstracción verbal entre los NNA rurales y urbanos, que no están siendo adecuadamente identificadas a través de esta subprueba o eventuales características en sus ítems (por ejemplo falta de familiaridad o pertinencia) o del proceso de administración (Analogías es la primera subprueba verbal que se aplica y requiere un alto nivel de dominio de procedimientos de administración por parte del evaluador/a) que pueden estar afectando el desempeño del los NNA de procedencia rural.

Estas posibles explicaciones, además del análisis de invarianza que puede evidenciar la presencia de sesgos de medición (Pedrero, 2019), alertan sobre la necesidad de revisar los ítems que componen la subprueba de Analogías a fin de determinar si existe o no, un funcionamiento diferencial (DIF) en sus reactivos que favorezca a los NNA de procedencia urbana por sobre los de procedencia rural.

A nivel de los factores de segundo orden los análisis realizados permiten sostener la presencia de invarianza métrica (considerando el comportamiento de la subprueba de Analogías en el paso anterior) indicando que estos cinco índices efectivamente dan cuenta de un factor de orden general, independientemente de la procedencia de los niños.

El hecho de no haber alcanzado un nivel de invarianza escalar sugiere que no es válido establecer comparaciones entre los rendimientos promedio de los niños de procedencia urbana y rural, en las diversas subpruebas que la componen, dado que los interceptos de Analogías y Búsqueda de Símbolos no serían equivalentes entre ambos grupos. Esto implica que un mejor desempeño en cualquiera de estas subpruebas no implica un mayor nivel de desarrollo en los factores latentes (Comprensión Verbal y Velocidad de Procesamiento, respectivamente).

A partir de los resultados obtenidos en esta investigación es posible sostener que a pesar de que la estructura factorial de la escala WISC-V es equivalente para ambos grupos, se observan diferencias a nivel de subpruebas que deben ser revisadas. Por tanto, se sugiere cautela especialmente en la interpretación de los puntajes de las subpruebas de Analogías y Búsqueda de Símbolos, cuando son aplicadas a niños de procedencia rural.

Finalmente, cabe destacar que los resultados de este estudio deben interpretarse considerando sus limitaciones. Dentro de estas, este trabajo sólo testeó la invarianza según la procedencia urbanarural por lo que sería recomendable que nuevas investigaciones incorporen otras fuentes de variación cultural como la etnia o género de los participantes. Por otra parte, dado que se utilizaron estrategias de muestreo no probabilístico, se limita la posibilidad de generalización de los resultados obtenidos, por lo que sería deseable su réplica en sectores rurales de otras regiones dentro de Chile, si se pretende alcanzar con esto una mayor representatividad. Por último, también puede ser considerada como una limitación de este estudio la falta de equivalencia entre los tamaños muestrales del grupo urbano y del rural, sin embargo fue necesario realizar los análisis estadísticos bajo esta estrategia considerando que estos no convergían en tamaños más reducidos de la muestra urbana, mientras que sí lo hacían en la rural.

\section{Referencias}

American Educational Research Association, American Psychological Association, \& National Council on Measurement in Education. (2014). Standards for educational and psychological testing. Washington, DC: American Educational Research Association.

Asociación Psicológica Americana (APA) (2010).

Enmiendas 2010, Recuperado de

http://www.psicologia.unam.mx/documentos/pdf/ comite_etica/Codigo_APA.pdf

Beaujean, A. A. (2016). Reproducing the Wechsler Intelligence Scale for Children-Fifth Edition: Factor model results. Journal of Psychoeducational Assessment, 34(4), 404408. doi:10.1177/0734282916642679

Brenlla, M. E. (2013). Interpretación del WISC- 
IV. Puntuaciones compuestas y modelos CHC. Ciencias Psicológicas, 7(2), 183-197.

Chen, F. (2007) Sensitivity of goodness of fit indexes to lack of measurement invariance. Structural equation modeling: A Multidisciplinary Journal, 14(3), 464-504. doi:10.1080/10705510701301834

Chen, H., Zhang, O., Raiford, S. E., Zhu, J., \& Weiss, L. G. (2015). Factor invariance between genders on the Wechsler Intelligence Scale for Children-Fifth Edition. Personality and Individual Differences, 86, 1-5. doi:10.1016/j.paid.2015.05.020

Chen H., Zhu J., Liao Y-K., \& Keith T. Z. (2020). Age and gender invariance in the Taiwan Wechsler Intelligence Scale for Children, Fifth Edition: Higher Order Five-Factor Model. Journal of Psychoeducational Assessment, 38(8), 1033-1045. doi: 10.1177/0734282920930542

Canivez, G. L., Watkins, M. W., \& Dombrowski, S. C. (2016). Factor structure of the Wechsler Intelligence Scale for Children-Fifth Edition: Exploratory factor analyses with the 16 primary and secondary subtests. Psychological Assessment, 28(8), 975-986. doi:10.1037/pas0000238

Canivez, G. L., Watkins, M. W., \& McGill, R. J. (2019). Construct validity of the Wechsler Intelligence Scale For Children - Fifth UK Edition: Exploratory and confirmatory factor analyses of the 16 primary and secondary subtests. British Journal of Educational Psychology, 89(2), 195-224. doi:10.1111/bjep.12230

Cianci, L. Orsini, A., Helbert, S., \& Pezutti, L. (2013). The influence of parents' education in the Italian standardization sample of the WISC-III. Learning \& Individual Differences, 28, 47-53. doi: 10.1016/j.lindif.2013.09.009

Coronel, P., Lacunza, B., \& Contini, N. (2006). Las habilidades cognitivas en niños privados culturalmente. Resultados preliminares de la primera fase de evaluación. Revista Iberoamericana de Diagnóstico y Evaluación - e Avaliação Psicológica, 2(22), 49-74.

Decreto No 170/09 (2009). Normativa Educación Especial. Ministerio de Educación de Chile. Recuperado de https://www.leychile.cl/Navegar?idNorma=10

\section{0}

Dimitrov, D. (2010). Testing for factorial invariance in the context of construct validation. Measurement and Evaluation in Counseling and Development, 43(2), 121-149. doi:10.1177/0748175610373459

Eilertsen, T., Thorsen, L., Holm, S. E., Bøe, T., Sørensen, L., \& Lundervold, A. (2016). Parental socioeconomic status and child intellectual functioning in a Norwegian sample. Scandinavian Journal of Psychology, 57(5), 399-405. doi:10.1111/sjop.12324

Elosua, P. (2005). Evaluación progresiva de la invarianza factorial entre las versiones original y adaptada de una escala de autoconcepto. Psicothema, 17(2), 356-362.

Flanagan, D., \& Alfonso, V. (2017). Essentials of WISC-V assessment. Hoboken, New Jersey, United States of America: Wiley.

Fenollar-Cortés, J., \& Watkins, M. W. (2019). Construct validity of the Spanish Version of the Wechsler Intelligence Scale for Children-Fifth Edition (WISC-V ${ }^{\text {Spain }}$ ). International Journal of School \& Educational Psychology, 7(3), 150-164. doi:10.1080/21683603.2017.1414006

Forns, M., \& Amador, J. (2017). Habilidades clínicas para aplicar, corregir e interpretar las escalas de inteligencia de Wechsler. Madrid, España: Pirámide.

Fuica, P., Lira, J., Alvarado, K., Araneda, C., Lillo, G., Miranda, R., \& Pérez-Salas, C. P. (2014). Habilidades cognitivas, contexto rural y urbano: Comparación de perfiles WAIS-IV en jóvenes. Terapia Psicológica, 32(2), 143152. doi: $10.4067 /$ S0718-48082014000200007

Hu, L. T., \& Bentler, P. M. (1999). Cutoff criteria for fit indexes in covariance structure analysis: Conventional criteria versus new alternatives. Structural Equation Modeling: A Multidisciplinary Journal, 6(1), 1-55. doi:10.1080/10705519909540118

Instituto Nacional de Estadísticas - Chile (2004). Chile: Estimaciones y proyecciones de población por sexo y edad. País urbano-rural. Santiago, Chile.

International Test Comission. (2017). ITC Guidelines for Translating and Adapting Tests (Second Edition), International Journal of Testing, 18(2), 101-134. 
doi:10.1080/15305058.2017.1398166

Kaufman, A. S., Raiford, S. E., \& Coalson, D. L. (2016). Intelligent testing with the WISC-V. (I. John Wiley \& Sons, Ed.) (First Edit). Hoboken, New Jersey, United States of America: Wiley.

Labin, A., Brenlla, M. A., \& Taborda, A. (2018). Índices alternativos del WISC-IV para la evaluación del razonamiento fluido. Revista Iberoamericana de Diagnóstico y Evaluación - e Avaliação Psicológica, 48(3), 81-90.

Labin, A., Taborda, A., \& Brenlla, M. (2015). La relación entre el nivel educativo de la madre y el rendimiento cognitivo infanto-juvenil a partir del WISC-IV. Psicogente, 18(34), 293302. doi:10.17081/psico.18.34.505

McGill, R., Ward, T., \& Canivez, G. (2020). Use of translated and adapted versions of the WISC-V: Caveat emptor. School Psychology International, 41(3) 276-294. doi: $10.1177 / 0143034320903790$

Ministerio de Educación, Centro de Estudios, Unidad de Estadísticas (2018). Estadísticas de la Educación 2017, Publicación diciembre 2018. Santiago, Chile.

Morris, J. D., Kelsey, E., \& Martin, R. A. (1980). Comparison of WISC-R performance of urban and rural special education students. Psychological Reports, 46(2), 671-677. doi:10.2466/pr0.1980.46.2.671

Niileksela, C. R., \& Reynolds, M. R. (2019). Enduring the tests of age and time: Wechsler constructs across versions and revisions. Intelligence, 77(c), 2-15. doi:10.1016/j.intell.2019.101403

Ordenanza N 05 de 2019 [División General de Educación]. Informa sobre nueva fecha de exigencia de uso de prueba WISC-V para evaluación diagnóstica en PIE en el marco del Decreto $\mathrm{N}^{\circ} 170.28$ de agosto de 2019. https://especial.mineduc.cl/wpcontent/uploads/sites/31/2019/11/ORD.-DEG1486-Informa-nueva-fecha-de-exigencia-deuso-de-prueba-WISC-V-Exp.-43897.pdf

Pauls, F., Daseking, M., \& Petermann, F. (2019). Measurement invariance across gender on the second-order five-factor model of the German Wechsler Intelligence Scale for Children-Fifth Edition. Assessment, 27(8), 1836-1852. doi:10.1177/1073191119847762.
Pedrero, V. (2019). Medición de invarianza factorial en pruebas de gran escala. En J. Manzi, M. García \& S. Taut (Eds.), Validez de las evaluaciones educacionales en Chile $y$ Latinoamérica (pp. 265-300). Santiago, Chile: Ediciones UC

Pérez, E. (2001). Hacia una nueva visión de lo rural. En: N. Giarracca (Comp.). ¿Una nueva ruralidad en América Latina? (pp. 17-29). CLACSO.

Putnick, D. L., \& Bornstein, M. H. (2016). Measurement invariance conventions and reporting: The state of the art and future directions for psychological research. Developmental Review, 41, 71-90. doi:10.1016/j.dr.2016.06.004

Ramírez, V., \& Rosas, R. (2007). Estandarización del WISC-III en Chile: Descripción del test, estructura factorial y consistencia interna de las escalas. Psykhe, 16(1), 91-109. doi:10.4067/S0718-22282007000100008

Reynolds, M. R., \& Keith, T. Z. (2017). Multigroup and hierarchical confirmatory factor analysis of the Wechsler Intelligence Scale for Children-Fifth Edition: What does it measure? Intelligence, 62, 31-47. doi:10.1016/j.intell.2017.02.005

Rodríguez-Cancino, M., Vizcarra, B., \& ConchaSalgado, A. (2020). Propiedades Psicométricas de la Escala WISC-V en escolares rurales chilenos [Manuscrito enviado a publicación]. Departamento de Psicología, Universidad de La Frontera.

Rodríguez, M., Rosas, R., \& Pizarro, M. (2019). (CEDETi UC Papeles de Investigación $\mathrm{N}^{\circ} 11$ ). Recuperado de http://www.cedeti.cl/investigacion-ydesarrollo/papeles-de-investigacion/

Rosas, R., \& Pizarro, M. (2018). WISC-V. Manual de Administración y Corrección. Santiago Chile. CEDETi-UC.

Rosas, R., Pizarro, M., Grez, O., Navarro; V., Tapia, D., Arancibia, S.,... von Fredeen, P. (en prensa). Estandarización chilena de la Escala Wechsler de Inteligencia para Niñosquinta edición. Psykhe

Scheiber, C. (2016). Is the Cattell-Horn-Carrollbased factor structure of the Wechsler Intelligence Scale for Children-Fifth Edition (WISC-V) construct invariant for a 
representative sample of African-American, Hispanic, and Caucasian male and female students ages 6 to 16 years? Journal of Pediatric Neuropsychology, 2, 79-88. doi:10.1007/s40817-016-0019-7

Smith, L. V., \& Graves, S. L. (2020). An exploration of gender invariance of the WISC$\mathrm{V}$ among black children in an urban school district. Contemporary School Psychology. doi:10.1007/s40688-020-00307-8

Tabachnick, B. G., \& Fidell, L. S. (2014). Using multivariate statistics (6th. Ed.). Harlow: Pearson Education Limited.

Treviño, E., Sandoval-Hernández, A., Miranda, D., Rutkowski, D., \& Matta, T. (2019). Invarianza de las escalas de nivel socioeconómico en estudios internacionales. En J. Manzi, M. García \& S. Taut (Eds.), Validez de las evaluaciones educacionales en Chile y Latinoamérica (pp. 302-328). Santiago, Chile: Ediciones UC

Van de Schoot, R., Lugtig, P., \& Hox, J. (2012). A checklist for testing measurement invariance. European Journal of Developmental Psychology, 9(4), 486-492. doi:10.1080/17405629.2012.686740

Vandenberg, R. J., \& Lance, C. E. (2000). A Review and synthesis of the measurement invariance literature: Suggestions, practices, and recommendations for organizational research. Organizational Research Methods, 3(1), 4-70. doi:10.1177/109442810031002

Van de Vijver, F., Weiss, L., Saklofske, D., Batty, A., \& Prifitera, A. (2019). A cross-cultural analysis of the WISC-V. En L.Weiss, D. Saklofke, J. Holdnack \& A. Prifitera. WISC-V. Clinical Use and Interpretation. Second Edition (pp. 223-244) London, United Kingdom: Academic Press. Elsevier

Wechsler, D. (2014). Wechsler Intelligence Scale for Children-Fifth Edition. Technical and Interpretive Manual. Bloomington, $\mathrm{MN}$ : Pearson Clinical Assessment.

Weiss, L., Saklofke, D., Holdnack, J., \& Prifitera, A. (2019). WISC-V. Clinical use and interpretation. Second Edition. Academic Press. Elsevier 